\title{
TOPOLOGICAL ENTROPY IN THE SYNCHRONIZATION OF PIECEWISE LINEAR AND MONOTONE MAPS. COUPLED DUFFING OSCILLATORS
}

\author{
ACILINA CANECO \\ Instituto Superior de Engenharia de Lisboa, Mathematics Unit, DEETC \\ and CIMA-UE, Rua Conselheiro Emidio Navarro, 1, 1949-014 Lisboa, \\ Portugal \\ E-mail: acilina@deetc.isel.ipl.pt \\ J. LEONEL ROCHA \\ Instituto Superior de Engenharia de Lisboa, Mathematics Unit, DEQ, and \\ CEAUL, Rua Conselheiro Emidio Navarro, 1, 1949-014 Lisboa, Portugal \\ E-mail: jrocha@deq.isel.ipl.pt \\ CLARA GRÁCIO \\ Department of Mathematics, Universidade de Évora and CIMA-UE, Rua \\ Romão Ramalho, 59, 7000-671 Évora, Portugal \\ E-mail:mgracio@uevora.pt
}

March 4, 2009

\begin{abstract}
In this paper is presented a relationship between the synchronization and the topological entropy. We obtain the values for the coupling parameter, in terms of the topological entropy, to achieve synchronization of two unidirectional and bidirectional coupled piecewise linear maps. In addition, we prove a result that relates the synchronizability of two m-modal maps with the synchronizability of two conjugated piecewise linear maps. An application to the unidirectional and bidirectional coupled identical chaotic Duffing equations is given. We discuss the complete synchronization of two identical double-well Duffing oscillators, from the point of view of symbolic dynamics. Working with Poincaré cross-sections and the return maps associated, the synchronization of the two oscillators, in terms of the coupling strength, is characterized. Keywords: Chaotic synchronization; topological entropy; Duffing oscillator; symbolic dynamics.
\end{abstract}

\section{Introduction}

Two or more oscillators are said to be coupled if they influence each other by any chemical or physical process. It has been observed that coupled systems starting from slightly different initial conditions would evolve in time, with completely different behaviour, but after some time they adjust a given property of their motion to a common behaviour. As described in [Pikovsky et al, 2001], synchronization is an adjustment of rhythms of oscillating objects due to their weak interaction. This phenomenon of oscillator's synchronization has been observed in nature like the fireflies, 
planets, pacemaker's cells, bridges and has been studied by mathematicians, physicists, biologists, astronomers, engineers and social biologists.

The coupling of two oscillators may be bidirectional, if each one influences the other, or unidirectional or master-slave if only one of the oscillators influence the other. If the coupled systems undergo a chaotic behaviour and they became synchronized, this is called a chaotic synchronization. Even the simplest system can display very complicated behaviour, but they can go chaotic in the same way. Chaotic synchronization is quite a rich phenomenon that may present several forms, like identical or complete synchronization, generalized synchronization, phase synchronization, anticipated synchronization, lag synchronization and amplitude envelope synchronization (see [Boccaletti et al, 2002], [Pikovsky et al, 2001] and [Gonzlez-Miranda, 2004]).

The analysis of synchronization phenomena of dynamical systems started in the 17th century with the finding of Huygens that two very weakly coupled pendulum clocks become synchronized in phase. Since then, several problems concerning the synchronization have been investigated, especially to know for what values of the coupling parameter there is synchronization. These values are, in general, given in terms of the function describing the local dynamics. If we have a network, i.e., there are more than two coupled systems, the synchronization interval depends not only on the local dynamics of the nodes, but also on the conexion topologie of the network and the synchronization interval may be bounded in both sides (see [Cao \& Lu, 2006]). In a previous work [Caneco et al, 2007] we study the synchronization and desynchronization threshold of a network, in terms of the topological entropy of each local node.

Duffing Equation has been the study object as a good example of rich chaotic behavior and also for the investigation of chaotic synchronization. In [Luo, 2008], is studied the mapping structures of chaos in the damped Duffing oscillator based on the traditional Poincaré mapping section and the switching plane defined on the separatrix (i.e. homoclinic or heteroclinic orbit). In [Kenfack, 2003], is studied the linear stability of the coupled double-well Duffing oscillators projected on a Poincaré section. In [Kyprianidis et al, 2006], is observed numerically the synchronization of two identical single-well Duffing oscillators. In [Vincent \& Kenfack, 2008], is studied numerically the bifurcation structure of a double-well Duffing oscillator coupled with a single-well one.

Symbolic dynamics is a fundamental tool available to describe complicated time evolution of a chaotic dynamical system. Instead of representing a trajectory by numbers, one uses sequences of symbols of a finite alphabet which symbols correspond to the intervals defined by the turning points of a map. We use symbolic dynamics methods to compute the topological entropy, with the goal to obtain a topological classification of the nonlinear oscillation behaviour for these $m$-modal maps (see [Rocha \& Ramos, 2006] and [Caneco et al, 2009]).

Complete synchronization is obtained when there is an identity between the trajectories of the two systems. In [Pecora \& Carroll, 1990] and [Pecora \& Carroll, 1991] it was establish that this kind of synchronization can be achieved provided that all the conditional Lyapunov exponents are negative. Since then, some authors [Shuai et al, 1997] have reported theirs computational experiments showing that apparently, it is possible to achieve synchronization without the negativity of all conditional Lyapunov exponents and some others (see [Cao \& Lu, 2006] and references there in) have reported that sometimes there is brief lack of synchronization in the region where all the conditional Lyapunov exponents are negative. It seems, that this is due to a numerical trap, because near the synchronization manifold, the two identical systems looks like in complete synchronization due to finite precision of numerical calculations (see [Pikovsky et al, 2001]).

The negativity of the conditional or transverse Lyapunov exponents is a necessary condition for the stability of the synchronized state (see [Boccaletti et al, 2002]) and is a mathematical expression of the decreasing to zero of the logarithm average of the distance of the solutions on the transverse manifold to the solutions on the synchronization manifold. So, if there is a strong convergence of this distance to zero, this average must decrease to zero. But the converse is not true. Even when all the conditional Lyapunov exponents 
are negative, it is possible that some orbits escape from the synchronization manifold, because this is only a weak synchronization, in the sense of Milnor (see [Pikovsky et al, 2001]). Only Lyapunov functions give necessary and sufficient conditions for the stability of the synchronization manifold.

Nevertheless, if the coupled systems are defined by piecewise linear maps, which is the case we are going to study in the next section, the weak and the strong concepts of synchronization coincides and it occurs for $k>k_{c}$. The synchronization threshold $k_{c}$ obtained from the assumption that all conditional Lyapunov exponents are negative, is expressed in terms of the Lyapunov exponent of the local map $f$. If this local map $f$ is a piecewise linear map with slope $\pm s$ everywhere, then its Lyapunov exponent is exactly $\log |s|$.

The layout of this paper is as follows: in section 2., we present the main result of this paper, we prove a theorem concerning conditions for the unidirectional and bidirectional synchronization of piecewise linear maps, in terms of the topological entropy and another theorem generalizing this result to piecewise monotone maps. In section 3., we investigate numerically the synchronization of two identical double-well Duffing oscillators, from the point of view of Symbolic Dynamics. First, in subsection 3.1., we consider the uncoupled case, searching regions in the parameter plane where the first return map defined by a Poincare section is like an unimodal or a bimodal map and we use Kneading Theory from Symbolic Dynamics, to evaluate the topological entropy for these $m$-modal maps, in subsection 3.2. Next, in subsection 3.3., we consider the coupling of chaotic Duffing equations, i.e., for parameter values for which the topological entropy is positive. Then, we find numerically the values of the coupling parameter for which there is chaotic synchronization. We consider the unidirectional and bidirectional coupling of Duffing oscillators and confirm the agreement of these observations with our theoretical results. Finally, in section 4., we discuss the relation between the synchronization of $m$-modal maps and the synchronization of the semi-conjugated piecewise linear maps whose existence is guaranteed by Milnor-Thurston theory.

\section{Synchronization and toplogical entropy for m-modal maps}

Consider a discrete dynamical system $u_{n+1}=f\left(u_{n}\right)$, where $u=\left(u_{1}, u_{2}, \ldots, u_{m}\right)$ is an $m$-dimensional state vector with $f$ defining a vector field $f: \mathbf{R}^{m} \rightarrow \mathbf{R}^{m}$. The coupling of two such identical maps $x_{n+1}=f\left(x_{n}\right)$ and $y_{n+1}=f\left(y_{n}\right)$ defines another discrete dynamical system $\varphi: \mathbf{N}_{0} \times \mathbf{R}^{2 m} \rightarrow \mathbf{R}^{2 m}$, i.e., $\varphi(0, x, y)=(x, y), \forall(x, y) \in \mathbf{R}^{2 m}$ and $\varphi(t+s, x, y)=\varphi(t, \varphi(s, x, y)), \forall(x, y) \in \mathbf{R}^{2 m}$, $\forall(t, s) \in \mathbf{N}_{0}^{2}$.

Denoting by $k$ the coupling parameter, if we consider an unidirectional coupling

$$
\left\{\begin{array}{l}
x_{n+1}=f\left(x_{n}\right) \\
y_{n+1}=f\left(y_{n}\right)+k\left[f\left(x_{n}\right)-f\left(y_{n}\right)\right]
\end{array},\right.
$$

then

$$
\varphi(n, x, y)=\left(f\left(x_{n}\right), f\left(y_{n}\right)+k\left[f\left(x_{n}\right)-f\left(y_{n}\right)\right]\right) .
$$

If the coupling is bidirectional

$$
\left\{\begin{array}{l}
x_{n+1}=f\left(x_{n}\right)-k\left[f\left(x_{n}\right)-f\left(y_{n}\right)\right] \\
y_{n+1}=f\left(y_{n}\right)+k\left[f\left(x_{n}\right)-f\left(y_{n}\right)\right]
\end{array},\right.
$$

then $\varphi(n, x, y)=\left(f\left(x_{n}\right)+k\left[f\left(y_{n}\right)-f\left(x_{n}\right)\right]\right.$, $\left.f\left(y_{n}\right)+k\left[f\left(x_{n}\right)-f\left(y_{n}\right)\right]\right)$.

These two systems are said to be in complete synchronization if there is an identity between the trajectories of the two systems, so we must look to the difference $z_{n}=y_{n}-x_{n}$ and see if this difference converges to zero, as $n \rightarrow \infty$. If the coupling is unidirectional then

$$
z_{n+1}=(1-k)\left[f\left(y_{n}\right)-f\left(x_{n}\right)\right] .
$$

If the coupling is bidirectional then

$$
z_{n+1}=(1-2 k)\left[f\left(y_{n}\right)-f\left(x_{n}\right)\right] .
$$

\subsection{Synchronization of piecewise linear maps}

Let $I=[a, b] \subseteq \mathbf{R}$ be a compact interval. By definition, a continuous map $f: I \rightarrow I$ which is piecewise monotone, i.e., there exist points $a=$ $c_{0}<c_{1}<\cdots c_{m}<c_{m+1}=b$ at which $f$ has a local extremum and $f$ is strictly monotone in each of the subintervals $I_{0}=\left[c_{0}, c_{1}\right], \ldots, I_{m}=\left[c_{m}, c_{m+1}\right]$, is called a $m$-modal map. As a particular case, if $f$ 
is linear in each subinterval $I_{0}, \ldots, I_{m}$, then $f$ is called a $m+1$ piecewise linear map. By theorem 7.4 from [Milnor \& Thurston, 1988] and [Parry, 1964] it is known that every $m$-modal map $f: I=[a, b] \subset$ $\mathbf{R} \rightarrow I$, with growth rate $s$ and positive topological entropy $h_{\text {top }}(f)\left(\log s=h_{\text {top }}(f)\right)$ is topologically semiconjugated to a $p+1$ piecewise linear map $T$, with $p \leq m$, defined on the interval $J=[0,1]$, with slope $\pm s$ everywhere and $h_{t o p}(T)=h_{t o p}(f)=\log s$, i.e., there exist a function $h$ continuous, monotone and onto, $h: I \rightarrow J$, such that $T \circ h=h \circ f$.

$$
\begin{array}{lllll} 
& & f & & \\
& I & \rightarrow & I & \\
& \downarrow & & \\
& \downarrow & & \downarrow & h \\
& J & \rightarrow & J & \\
& & T & &
\end{array}
$$

If, in addition, $h$ is a homeomorphism, then $f$ and $T$ are said topologically conjugated.

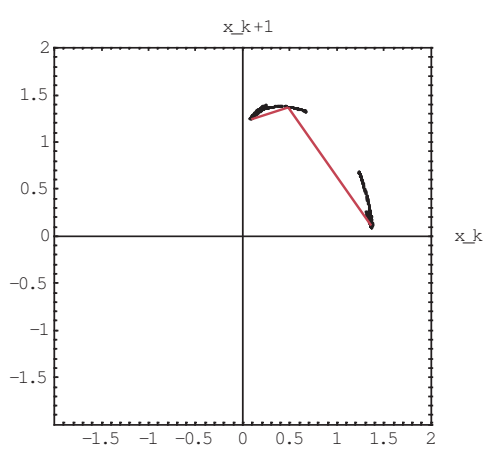

Fig. 1. Semiconjugacy between piecewise monotone and piecewise linear unimodal maps

According to the above statements, we will investigate the synchronization of two identical $p+1$ piecewise linear maps with slope $\pm s$ everywhere (Theorem 2.1.) and also the synchronization of two identical $m$-modal maps (Theorem 2.2.).

In what follows we will use the symbols $f$ and $k$ to represent, respectively, the $m$-modal map and its coupling parameter and the symbols $T$ and $c$ to represent, respectively, the $p+1$ piecewise linear map and its coupling parameter.

Let $T: J=\left[a_{1}, b_{1}\right] \subseteq \mathbf{R} \rightarrow J$, be a continuous piecewise linear map, i.e., there exist points $a_{1}=$ $d_{0}<d_{1}<\cdots d_{p}<d_{p+1}=b_{1}$ such that $T$ is linear in each subintervals $J_{i}=\left[d_{i}, d_{i+1}\right],(i=0, \ldots, p)$, with slope $\pm s$ everywhere.

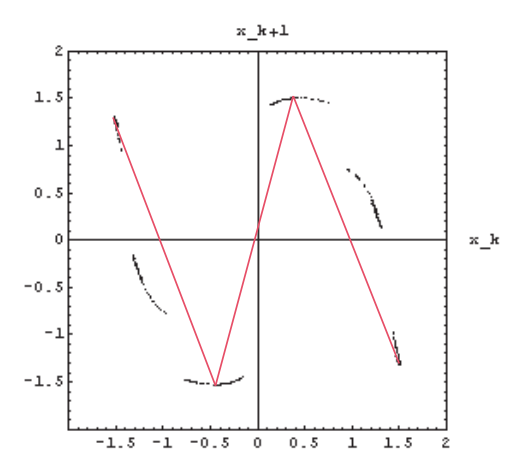

Fig. 2. Semiconjugacy between piecewise monotone and piecewise linear bimodal maps

So, the unidirectional coupled system for $T$ is

$$
\left\{\begin{array}{l}
X_{n+1}=T\left(X_{n}\right) \\
Y_{n+1}=T\left(Y_{n}\right)+c\left[T\left(X_{n}\right)-T\left(Y_{n}\right)\right]
\end{array},\right.
$$

and the difference $Z_{n}=Y_{n}-X_{n}$ verifies

$$
Z_{n+1}=(1-c)\left[T\left(Y_{n}\right)-T\left(X_{n}\right)\right] .
$$

For the bidirectionally coupled system

$$
\left\{\begin{array}{rl}
X_{n+1} & =T\left(X_{n}\right)-c\left[T\left(X_{n}\right)-T\left(Y_{n}\right)\right] \\
Y_{n+1} & =T\left(Y_{n}\right)+c\left[T\left(X_{n}\right)-T\left(Y_{n}\right)\right]
\end{array},\right.
$$

the difference $Z_{n}=Y_{n}-X_{n}$ verifies

$$
Z_{n+1}=(1-2 c)\left[T\left(Y_{n}\right)-T\left(X_{n}\right)\right]
$$

Theorem 2.1. Let $T: J \rightarrow J$, be a continuous $p+1$ piecewise linear map with slope $\pm s$ everywhere, with $s>1$. Let $c \in[0,1]$ be the coupling parameter. Then one has:

(i) The unidirectional coupled system (5) is synchronized if

$$
c>\frac{s-1}{s}
$$

(ii) The bidirectional coupled system (7) is synchronized if

$$
\frac{s+1}{2 s}>c>\frac{s-1}{2 s} .
$$


Proof. Attending to the fact that $T$ is linear with slope $\pm s$ in each subinterval $J_{0}, \ldots, J_{p}$, then, the total variation of $T$ is

$$
\begin{aligned}
V_{b_{1}}^{a_{1}}(T) & =\int_{a_{1}}^{b_{1}}\left|T^{\prime}(t)\right| d t=\sum_{i=0}^{p} \int_{d_{i}}^{d_{i+1}}\left|T^{\prime}(t)\right| d t \\
& =s \sum_{i=0}^{p}\left|d_{i+1}-d_{i}\right|=s\left|b_{1}-a_{1}\right| .
\end{aligned}
$$

We have

$$
\begin{aligned}
\left|T\left(Y_{n}\right)-T\left(X_{n}\right)\right| & =\left|\int_{X_{n}}^{Y_{n}} T^{\prime}(t) d t\right| \leq \int_{X_{n}}^{Y_{n}}\left|T^{\prime}(t)\right| d t \\
& =V_{Y_{n}}^{X_{n}}(T)=s\left|Y_{n}-X_{n}\right| .
\end{aligned}
$$

Attending to (6), it follows that,

$$
\left|Z_{n+1}\right| \leq|(1-c) s|\left|Z_{n}\right|
$$

and then

$$
\left|Z_{q}\right| \leq|(1-c) s|^{q}\left|Z_{0}\right| .
$$

So, letting $q \quad \rightarrow \quad \infty$, we have $\lim _{q \rightarrow+\infty}|(1-c) s|^{q}\left|Z_{0}\right|=0$, if $|(1-c) s|<1$. The previous arguments shows that, if $c \in[0,1]$ then the unidirectional coupled system (5) is synchronized if $c>\frac{s-1}{s}$.

On the other hand, using the same arguments as before and attending to (8), we have

$$
\left|Z_{n+1}\right| \leq|(1-2 c) s|\left|Z_{n}\right|
$$

and then

$$
\left|Z_{q}\right| \leq|(1-2 c) s|^{q}\left|Z_{0}\right| .
$$

Thus, considering $q \rightarrow \infty$, we have $\lim _{q \rightarrow+\infty}|(1-2 c) s|^{q}\left|Z_{0}\right|=0$, if $|(1-2 c) s|<1$. Therefore, we may conclude that, if $c \in[0,1]$ the bidirectional coupled system (8) is synchronized if $\frac{s+1}{2 s}>c>\frac{s-1}{2 s}$.

Note that, the bidirectional synchronization occurs at half the value of the coupling parameter for the unidirectional case, as mentioned in [Belykh et al, 2007].

\subsection{Synchronization of two piecewise monotone maps}

In this section our question is to know the relationship between the synchronization of two coupled identical $m$-modal maps and the synchronization of the two coupled corresponding conjugated $p+1$ piecewise linear maps, with $p \leq m$. Consider in the interval $J$ the pseudometric defined by

$$
d(x, y)=|h(x)-h(y)| .
$$

If $h$ is only a semiconjugacy, $d$ is not a metric because one may have $d(x, y)=0$ for $x \neq y$. Nevertheless, if $h$ is a conjugacy, then the pseudometric $d$, defined above, is a metric. Two metrics $d_{1}$ and $d_{2}$ are said to be topologically equivalent if they generate the same topology. A sufficient but not necessary condition for topological equivalence is that for each $x \in I$, there exist constants $k_{1}, k_{2}>0$ such that, for every point $y \in I$,

$$
k_{1} d_{1}(x, y) \leq d_{2}(x, y) \leq k_{2} d_{1}(x, y) .
$$

Consider the pseudometric $d$ defined above, $d_{2}(x, y)=d(x, y)$ and $d_{1}(x, y)=|x-y|$.

Suppose $h: I \rightarrow J$ is a bi-Lipschitz map, i.e., $\exists N, M>0$, such that,

$0<N|x-y| \leq|h(x)-h(y)| \leq M|x-y|, \quad \forall(x, y) \in I^{2}$.

If $h$ is a conjugacy and verifies (9), then the metrics $d$ and |.| are equivalents.

Let $f: I[a, b] \subset \mathbf{R} \rightarrow I$ be a function $m$-modal, with positive entropy. For the unidirectional coupled system given by (1) we have the difference (3). As for the bidirectional coupled system given by (2) we have the difference (4).

As an extension of Theorem 2.1., for the synchronization of piecewise linear maps, we can establish the following result concerning the synchronization of the corresponding semiconjugated piecewise monotone maps.

Theorem 2.2. Let $f: I \rightarrow I$, be a continuous and piecewise monotone map with positive topological entropy $h_{\text {top }}=\log s$ and $h: I \rightarrow J$ a semiconjugacy between $f$ and a continuous piecewise linear map $T: J \rightarrow J$, with slope $\pm s$ everywhere. If there exist constants $N, M>0$ satisfying (9), then one has: 
(i) The unidirectional coupled system (1) is synchronized if

$$
k>1-\frac{N}{M} \frac{1}{s} .
$$

(ii) The bidirectional coupled system (2) is synchronized if

$$
1+\frac{N}{M} \frac{1}{2 s}>k>1-\frac{N}{M} \frac{1}{2 s} .
$$

Proof. If $f$ is monotone in the interval $[x, y]$, then $T$ is monotone in the interval $[h(x), h(y)]$, because $h$ is monotone, so

$$
\begin{aligned}
|h(f(x))-h(f(y))| & =|T(h(x))-T(h(y))| \\
& =s|h(x)-h(y)| .
\end{aligned}
$$

Therefore $d(x, y)=s^{-1} d(f(x), f(y))$, if $f$ is monotone in the interval $[x, y]$. If $f$ is not monotone in the interval $[x, y]$, but there exist, points $c_{i}(i=$ $1, \ldots, p-1)$, such that $c_{i}<c_{i+1}, c_{i} \in[x, y]$ and $f$ is monotone in each subinterval $I_{1}=\left[x=c_{0}, c_{1}\right]$, $I_{2}=\left[c_{1}, c_{2}\right], \ldots, I_{p}=\left[c_{p-1}, y=c_{p}\right]$, we have

$$
\begin{aligned}
d(x, y) & =\sum_{j=0}^{p-1} d\left(c_{j}, c_{j+1}\right) \\
& =s^{-1} \sum_{j=0}^{p-1} d\left(f\left(c_{j}\right), f\left(c_{j+1}\right)\right) \\
& =s^{-1} \sum_{j=0}^{p-1}\left|h\left(f\left(c_{j}\right)\right)-h\left(f\left(c_{j+1}\right)\right)\right| \\
& \geq s^{-1}|h(f(x))-h(f(y))| \\
& =s^{-1} d(f(x), f(y)) .
\end{aligned}
$$

So, we can write $d(x, y) \geq s^{-1} d(f(x), f(y))$, $\forall x, y \in I$. From (9) and for the unidirectional coupling (3) we have

$$
\begin{aligned}
d\left(y_{n+1}, x_{n+1}\right) & \leq M\left|y_{n+1}-x_{n+1}\right| \\
& =M|1-k|\left|f\left(y_{n}\right)-f\left(x_{n}\right)\right| \\
& \leq M|1-k| N^{-1} d\left(f\left(y_{n}\right), f\left(x_{n}\right)\right) \\
& \leq M|1-k| N^{-1} s d\left(y_{n}, x_{n}\right) .
\end{aligned}
$$

It follows that

$$
d\left(y_{n+r}, x_{n+r}\right) \leq M^{r}|1-k|^{r} N^{-r} s^{r} d\left(y_{n}, x_{n}\right),
$$

so if $\left|M(1-k) N^{-1} s\right|<1, d\left(y_{n+r}, x_{n+r}\right) \rightarrow 0$ as $r \rightarrow$ $\infty$.
Then, the coupled system (1) is synchronized if

$$
k>1-\frac{N}{M} \frac{1}{s} .
$$

For the bidirectional coupling (4) and using the same arguments as before, we also have that

$d\left(y_{n+1}, x_{n+1}\right) \leq M|1-2 k| N^{-1} s d\left(y_{n}, x_{n}\right)$.

It follows that $d\left(y_{n+r}, x_{n+r}\right) \rightarrow 0$ as $r \rightarrow \infty$, if $\left|M(1-2 k) N^{-1} s\right|<1$. Then, the coupled system (2) is synchronized if

$$
\frac{1}{2}\left(1+\frac{N}{M} \frac{1}{s}\right)>k>\frac{1}{2}\left(1-\frac{N}{M} \frac{1}{s}\right)
$$

Denote by $k^{*}$ the synchronization threshold for (1), i.e. the system of piecewise monotone functions synchronizes for $k>k^{*}$. Denote by $c^{*}$ the value such that for $c>c^{*}$ the system of piecewise linear maps (5) is synchronized. Note that

$$
N\left(1-k^{*}\right)=M\left(1-c^{*}\right) .
$$

With the assumptions we made, if the piecewise monotone coupled maps synchronizes, so do the conjugated piecewise linear coupled maps and conversely, if the piecewise linear coupled maps synchronizes, so do the conjugated piecewise monotone coupled maps. In fact, from (9) we have $d\left(y_{n}, x_{n}\right) \leq M\left|y_{n}-x_{n}\right|$, therefore if system (1) synchronizes for $k>k^{*}$, then system (5) synchronizes for $c>c^{*}$, because $k^{*} \geq c^{*}$. On the other hand, we have also from (9), $\left|y_{n}-x_{n}\right| \leq N^{-1} d\left(y_{n}, x_{n}\right)$, therefore if the system (5) synchronizes for $c>c^{*}$, then the system (1) synchronizes for $k>k^{*}$ with $k^{*}$ verifying (10).

For the bidirectional coupling, we have

$$
1-\frac{1}{s} \leq 1-\frac{N}{M} \frac{1}{s} \leq 1+\frac{N}{M} \frac{1}{s} \leq 1+\frac{1}{s},
$$

so the synchronization interval for the piecewise monotone coupled maps is contained in the synchronization interval for the conjugated piecewise linear coupled maps. 


\section{Duffing application}

In this section we test the above theoretical results in the Duffing oscillators. First we study the uncoupled case, choosing parameter values for which the Duffing equation exhibits chaotic behavior. In order to know that there is chaos we compute the topological entropy, using methods from Symbolic Dynamics, see [Caneco et al, 2009] [Rocha \& Ramos, 2006]. Then, we consider the unidirectional and bidirectional coupling of two chaotic Duffing equations and find numerically the values of the coupling parameter for which there is synchronization. In this case this is chaotic synchronization.

\subsection{The uncoupled case. Unimodal and bimodal maps in the Poincaré section}

Consider a periodically forced, damped Duffing oscillator with a twin-well potential defined by

$$
x^{\prime \prime}(t)=x(t)-x^{3}(t)-\alpha x^{\prime}(t)+\beta \cos (w t),
$$

where the parameters $\beta$ and $w$ are the excitation strength and frequency of the periodic loading, respectively [Luo, 2008]. In the particular case of the undamped $(\alpha=0)$ and the unforced $(\beta=0)$ one can obtain an exact solution of this non-linear second-order differential equation. Duffing equation is a classical example of a dynamical system that exhibits chaotic behavior. Attending to the complexity of the above equation, a basic tool is to do an appropriate Poincaré section to reduce the dimensionality. In our case, we did a section defined by $y=0$, since it is transversal to the flow, it contains all fixed points and captures most of the interesting dynamics. In order to see how the first return Poincaré map change with the parameters we did bifurcation diagrams. See in Fig. 3 the variation of the coordinate $x_{n}$ of the first return Poincaré map, versus the parameter $\beta \in[0.15,0.5]$, for a fixed value of $\alpha=0.25$. It is clear the growing of complexity as the parameter $\beta$ increases.

Consider parameter values and initial conditions for which each uncoupled system exhibits a chaotic behaviour and its first return Poincaré map is like a unimodal or like a bimodal map. Fixing, for example,

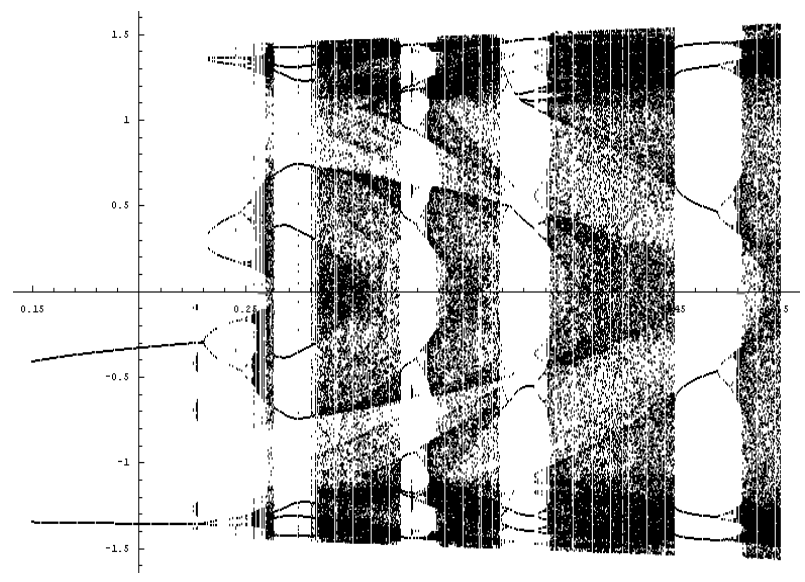

Fig. 3. Bifurcation diagram for $x_{n}$ as a function of $\beta \in[0.15,0.5]$, for a fixed $\alpha=0.25$.

$w=1.18, x_{0}=0.5, x_{0}^{\prime}=-0.3, y_{0}=0.9$, $y_{0}^{\prime}=-0.2$, we choose $(\alpha, \beta)=(0.2954,0.2875)$ and $(\alpha, \beta)=(0.25,0.2541)$, for the unimodal case (see Fig. 4 and Fig. 5) and $(\alpha, \beta)=(0.5,0.719)$ and $(\alpha, \beta)=(0.25,0.4998)$, for the bimodal case (see Fig. 6 and Fig. 7).
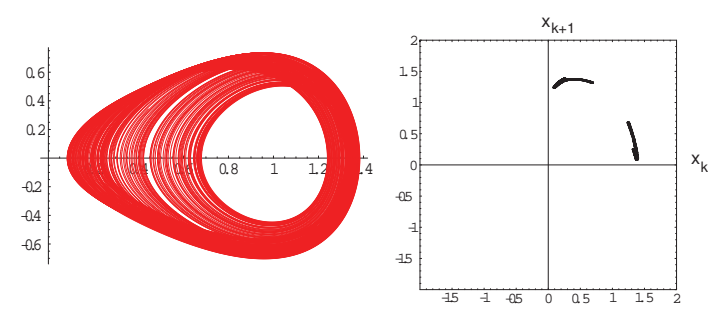

Fig. 4. Duffing attractor and Poincaré return map for $\alpha=0.2954$ and $\beta=0.2875$.

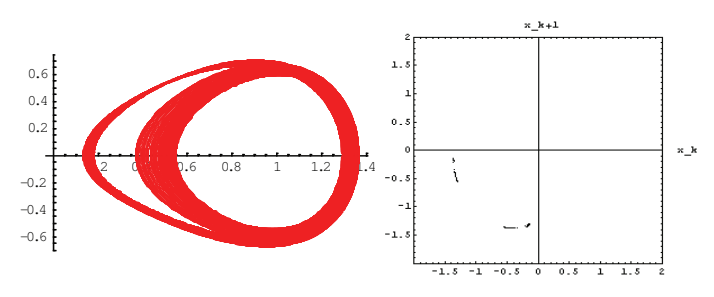

Fig. 5. Duffing attractor and Poincaré return map for $\alpha=0.25$ and $\beta=0.2541$.

We found in the parameter plane $(\alpha, \beta)$, a region $\mathcal{U}$ where the first return Poincaré map behaves like a unimodal map and a region $\mathcal{B}$ where 

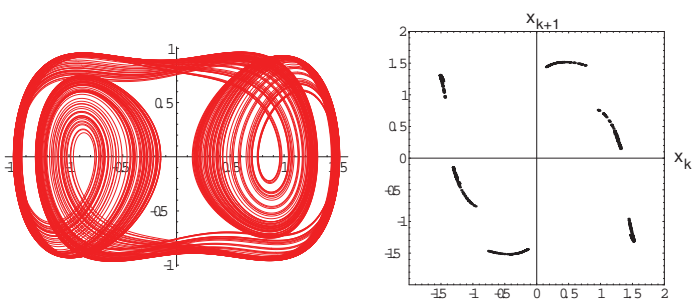

Fig. 6. Duffing attractor and Poincaré return map for $\alpha=0.5$ and $\beta=0.719$.

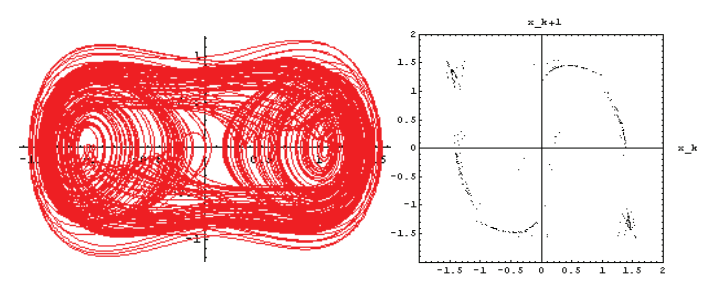

Fig. 7. Duffing attractor and Poincaré return map for $\alpha=0.25$ and $\beta=0.4998$.

the first return Poincaré map behaves like a bimodal map, see Fig. 8.

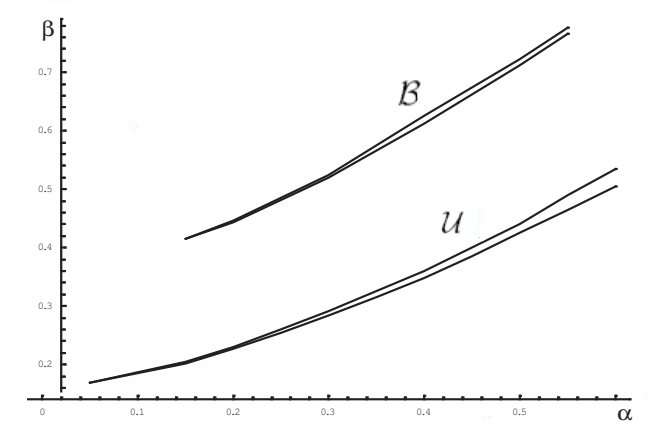

Fig. 8. Unimodal $\mathcal{U}$ and bimodal $\mathcal{B}$ regions in the parameter $(\alpha, \beta)$ plane.

\subsection{Entropy evaluation by kneading theory}

Consider a compact interval $I \subset R$ and a $m$-modal map $f: I \rightarrow I$, i.e., the map $f$ is piecewise monotone, with $m$ critical points and $m+1$ subintervals of monotonicity. Suppose $I=\left[c_{0}, c_{m+1}\right]$ can be divided by a partition of points $\mathcal{P}=\left\{c_{0}, c_{1}, \ldots, c_{m+1}\right\}$ in a finite number of subintervals $I_{1}=\left[c_{0}, c_{1}\right], \quad I_{2}=\left[c_{1}, c_{2}\right]$, $\ldots \quad, I_{m+1}=\left[c_{m}, c_{m+1}\right]$, in such a way that the restriction of $f$ to each interval $I_{j}$ is strictly monotone, either increasing or decreasing. Assuming that each interval $I_{j}$ is the maximal interval where the function is strictly monotone, these intervals $I_{j}$ are called laps of $f$ and the number of distinct laps is called the lap number, $\ell$, of $f$. In the interior of the interval $I$ the points $c_{1}, c_{2}, \ldots, c_{m}$, are local minimum or local maximum of $f$ and are called turning or critical points of the function. The limit of the $n$-root of the lap number of $f^{n}$ (where $f^{n}$ denotes the composition of $f$ with itself $n$ times) is called the growth number of $f$, i.e., $s=$ $\lim _{n \rightarrow \infty} \sqrt[n]{\ell\left(f^{n}\right)}$. In [Misiurewicz \& Szlenk, 1980] is defined the topological entropy as the logarithm of the growth number $h_{t o p}(f)=\log s$. In [Milnor \& Thurston, 1988] is developed the concept of kneading determinant, denoted by $D(t)$, as a formal power series from which we can compute the topological entropy as the logarithm of the inverse of its minimum real positive root. On the other hand, in [Lampreia \& Ramos, 1997], is proved, using homological properties, a precise relation between the kneading determinant and the characteristic polynomial of the Markov transition matrix associated with the itinerary of the critical points. In fact, they proved that the topological entropy is the logarithm of the spectral radius of this matrix.

The intervals $I_{j}=\left[c_{j-1}, c_{j}\right]$ are separated by the critical points, numbered by its natural order $c_{1}<c_{2}<\ldots<c_{m}$. We compute the images by $f$, $f^{2}, \ldots, f^{n}, \ldots$ of a critical point $c_{j}(j=1, \ldots, m-1)$ and we obtain its orbit

$$
O\left(c_{j}\right)=\left\{c_{j}^{n}: c_{j}^{n}=f^{n}\left(c_{j}\right), n \in N\right\} .
$$

If $f^{n}\left(c_{j}\right)$ belongs to an open interval $\left.I_{k}=\right] c_{k-1}, c_{k}[$, then we associate to it a symbol $L_{k}$, with $k=$ $1, \ldots, m+1$. If there is an $r$ such that $f^{n}\left(c_{j}\right)=c_{r}$, with $r=1, \ldots, m$, then we associate to it the symbol $A_{r}$. So, to each point $c_{j}$, we associate a symbolic sequence, called the address of $f^{n}\left(c_{j}\right)$, denoted by $S=S_{1} S_{2} \ldots S_{n} \ldots$, where the symbols $S_{k}$ belong to the $m$-modal alphabet, with $2 m+1$ symbols, i.e., $\mathcal{A}_{m}=\left\{L_{1}, A_{1}, L_{2}, A_{2}, \ldots, A_{m}, L_{m+1}\right\}$. The symbolic sequence $S=S_{0} S_{1} S_{2} \ldots S_{n} \ldots$, can be periodic, eventually periodic or aperiodic 
[Rocha \& Ramos, 2006]. The address of a critical point $c_{j}$ is said eventually periodic if there is a number $p \in N$, such that the address of $f^{n}\left(c_{j}\right)$ is equal to the address of $f^{n+p}\left(c_{j}\right)$, for large $n \in N$. The smallest of such $p$ is called the eventual period.

To each symbol $L_{k} \in \mathcal{A}_{m}$, with $k=1, \ldots, m+1$, define its sign by

$$
\varepsilon\left(L_{k}\right)=\left\{\begin{aligned}
-1 & \text { if } f \text { is decreasing in } I_{k} \\
1 & \text { if } f \text { is increasing in } I_{k}
\end{aligned}\right.
$$

and $\varepsilon\left(A_{k}\right)=0$, with $k=1, \ldots, m$. We can compute the numbers $\tau_{k}=\prod_{i=0}^{k-1} \varepsilon\left(L_{k}\right)$ for $k>0$, and take $\tau_{0}=1$. The invariant coordinate of the symbolic sequence $S$, associated with a critical point $c_{j}$, is defined as the formal power series

$$
\theta_{c_{j}}(t)=\sum_{k=0}^{k=\infty} \tau_{k} t^{k} S_{k} .
$$

The kneading increments of each critical point $c_{j}$ are defined by

$$
\nu_{c_{j}}(t)=\theta_{c_{j}^{+}}(t)-\theta_{c_{j}^{-}}(t) \quad \text { with } \quad j=1, \ldots m,
$$

where $\theta_{c_{j}^{ \pm}}(t)=\lim _{x \rightarrow c_{j}^{ \pm}} \theta_{x}(t)$. Separating the terms associated with the symbols $L_{1}, L_{2}, \ldots, L_{m+1}$ of the alphabet $\mathcal{A}_{m}$, the increments $\nu_{j}(t)$, are written in the form

$\nu_{c_{j}}(t)=N_{j 1}(t) L_{1}+N_{j 2}(t) L_{2}+\ldots+N_{j(m+1)}(t) L_{m+1}$.

The coefficients $N_{j k}$ in the ring $Z[[t]]$ are the entries of the $m \times(m+1)$ kneading matrix

$$
N(t)=\left[\begin{array}{ccc}
N_{11}(t) & \cdots & N_{1(m+1)}(t) \\
\vdots & \ddots & \vdots \\
N_{m 1}(t) & \cdots & N_{m(m+1)}(t)
\end{array}\right] .
$$

From this matrix we compute the determinants $D_{j}(t)=\operatorname{det} \widehat{N}(t)$, where $\widehat{N}(t)$ is obtained from $N(t)$ removing the $j$ column $(j=1, \ldots, m+1)$, and

$$
D(t)=\frac{(-1)^{j+1} D_{j}(t)}{1-\varepsilon\left(L_{j}\right) t}
$$

is called the kneading determinant. Here $\varepsilon\left(L_{j}\right)$ is defined like in (12).

Let $f$ be a $m$-modal map and $D(t)$ defined as above. Let $s$ be the growth number of $f$, then the topological entropy of the map $f$ is, see [Milnor \& Thurston, 1988],

$$
h_{\text {top }}(f)=\log s, \quad \text { with } \quad s=\frac{1}{t^{*}} .
$$

and

$$
t^{*}=\min \{t \in[0,1]: D(t)=0\}
$$

Let's take the Duffing equation (11) with the parameter values $\alpha=0.2954$ and $\beta=0.2875$. In this case the attractor and the unimodal Poincaré return map are shown in Fig. 4.

The symbolic sequence is $(C R L R R R)^{\infty}$, so we have:

$$
c^{+} \rightarrow(R R L R R R)^{\infty} \text { and } c^{-} \rightarrow(L R L R R R)^{\infty} .
$$

The invariant coordinates of the sequence $S$ associated with the critical point $c$ are

$$
\begin{gathered}
\theta_{c^{+}}(t)=\frac{t^{2}}{1+t^{6}} L+\frac{1-t+t^{3}-t^{4}+t^{5}}{1+t^{6}} R \\
\theta_{c^{-}}(t)=\frac{1-t^{2}}{1-t^{6}} L+\frac{t-t^{3}+t^{4}-t^{5}}{1-t^{6}} R .
\end{gathered}
$$

The kneading increment of the critical point, $\nu_{c}(t)=\theta_{c^{+}}(t)-\theta_{c^{-}}(t)$, is

$$
\begin{aligned}
\nu_{c}(t)= & \frac{-1+2 t^{2}-t^{6}}{1-t^{12}} L \\
& +\frac{1-2 t+2 t^{3}-2 t^{4}+2 t^{5}-t^{6}}{1-t^{12}} R .
\end{aligned}
$$

So, the kneading matrix is $N(t)=$ $\left[N_{11}(t) \quad N_{12}(t)\right]=\left[\begin{array}{ll}D_{2}(t) & D_{1}(t)\end{array}\right]$, with

$$
N_{11}(t)=\frac{-1+2 t^{2}-t^{6}}{1-t^{12}}
$$

and

$$
N_{12}=\frac{1-2 t+2 t^{3}-2 t^{4}+2 t^{5}-t^{6}}{1-t^{12}} .
$$

The kneading determinant is

$$
D(t)=\frac{(-1+t)\left(-1+t^{2}+t^{4}\right)}{1-t^{12}} .
$$

The smallest positive real root of $D_{1}(t)$ is $t^{*}=$ $0.786 \ldots$, so the growth number is $s=1 / t^{*}=1.272 \ldots$ and the topological entropy is $h_{t o p}=0.2406 \ldots$.

By this method, we compute some values of the topological entropy $h_{t o p}$ for other values of the coupling parameter $k$ for the unimodal and for the bimodal maps. See in Fig. 11 some examples of the entropy for fixed $\alpha=0.4$ and varying $\beta$, showing the growing of complexity. 


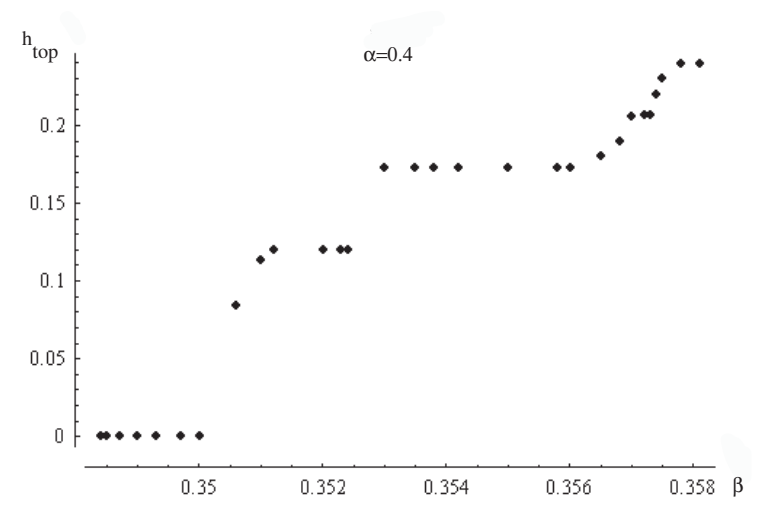

Fig. 9. Evolution of the topological entropy for the uncoupled Duffing equation, fixing $\alpha=0.4$ and varying $\beta$.

\subsection{Numerical synchronization of two identical Duffing oscillators}

Consider two identical unidirectionally coupled Duffing oscillators

$$
\left\{\begin{aligned}
x^{\prime \prime}(t)= & x(t)-x^{3}(t)-\alpha x^{\prime}(t)+\beta \cos (w t) \\
y^{\prime \prime}(t)= & y(t)-y^{3}(t)-\alpha y^{\prime}(t)+k[x(t)-y(t)] \\
& +\beta \cos (w t)
\end{aligned}\right.
$$

where $k$ is the coupling parameter, see [Vincent \& Kenfack, 2008] and references therein. We will choose parameter values for which each uncoupled oscillator exhibits a chaotic behaviour, so if they synchronize, that will be a chaotic synchronization.

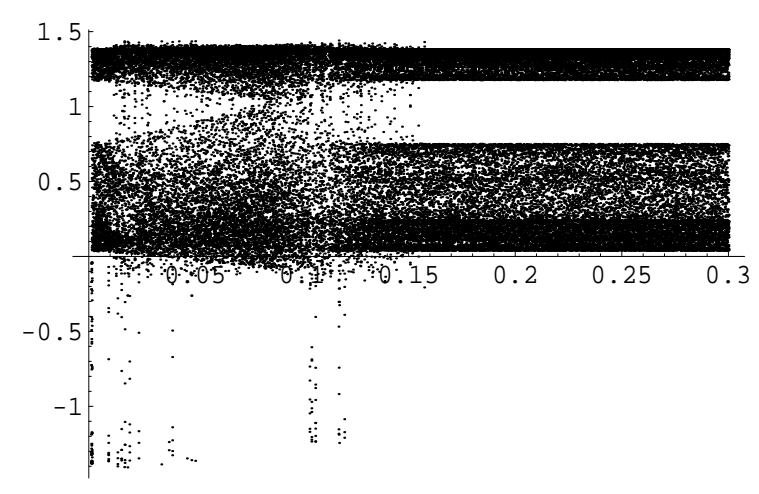

Fig. 10. Bifurcation diagram for $x_{n}$ as a function of $k \in[0.001,0.3]$, for fixed values of $\alpha=0.4$ and $\beta=0.3578$.
In Fig. 10 the bifurcation diagram for the unidirectional coupled system (19) with $\alpha=0.4$, $\beta=0.3578$ and the coupling parameter, $k \in$ $[0.001,0.03]$, shows several kind of regions. In Table 1, we show the topological entropy in some points of these regions. We choose for example the values $k=0, k=0.05, k=0.13, k=$ 0.5 and $k=0.301$. We verify that for $k$ larger than $k \approx 0.13$ the topological entropy remains constant, but positive. Meanwhile we find values, of the $k$ parameter, where the entropy is zero, that is, where there is chaos-destroying synchronization, see [Pikovsky et al, 2001]. In a previous work [Caneco et al, 2008] we try to understand the relationship between the achievement of synchronization and the evolution of the symbolic sequences $S_{x}$ and $S_{y}$, obtained for the $x$ and $y$ coordinates, as described in subsection 3.2 .. We verify that, as the value of the coupling parameter $k$ increases, the number of initial equal symbols in the $S_{x}$ and $S_{y}$ sequences increases also, which is a numerical symbolic evidence that the two systems are synchronized.

Notice the correspondence of these values for the topological entropy with the evolution of $k$ in the bifurcation diagram, see Fig. 10. Numerically we can also see the evolution of the difference $z=y-x$ with $k$. The synchronization will occur when $x=y$. See some examples in Fig. 11 for the unimodal case and in Fig. 12 for the bimodal case.

Although not shown in this figure, the graphics of the difference $y-x$ for $k$ greater then 0.122 are always a diagonal like in the picture for $k=0.25$, showing that these Poincaré unimodal maps are synchronized. Notice that, the pictures in Fig.11 and Fig.12 confirms numerically the theoretical results given by the above theorems. For $\alpha=0.4$ and $\beta=0.3578$ (Fig.11) which correspond to $s=$ $1.272 \ldots$, the synchronization occurs for $k>0.214 \ldots$ For the bimodal case, $\alpha=0.5$ and $\beta=0.719$, (Fig.12), which correspond to $s=2,618 \ldots$, the synchronization occurs for $k>0.873 \ldots$.

Consider the bidirectional coupled Duffing oscillators 

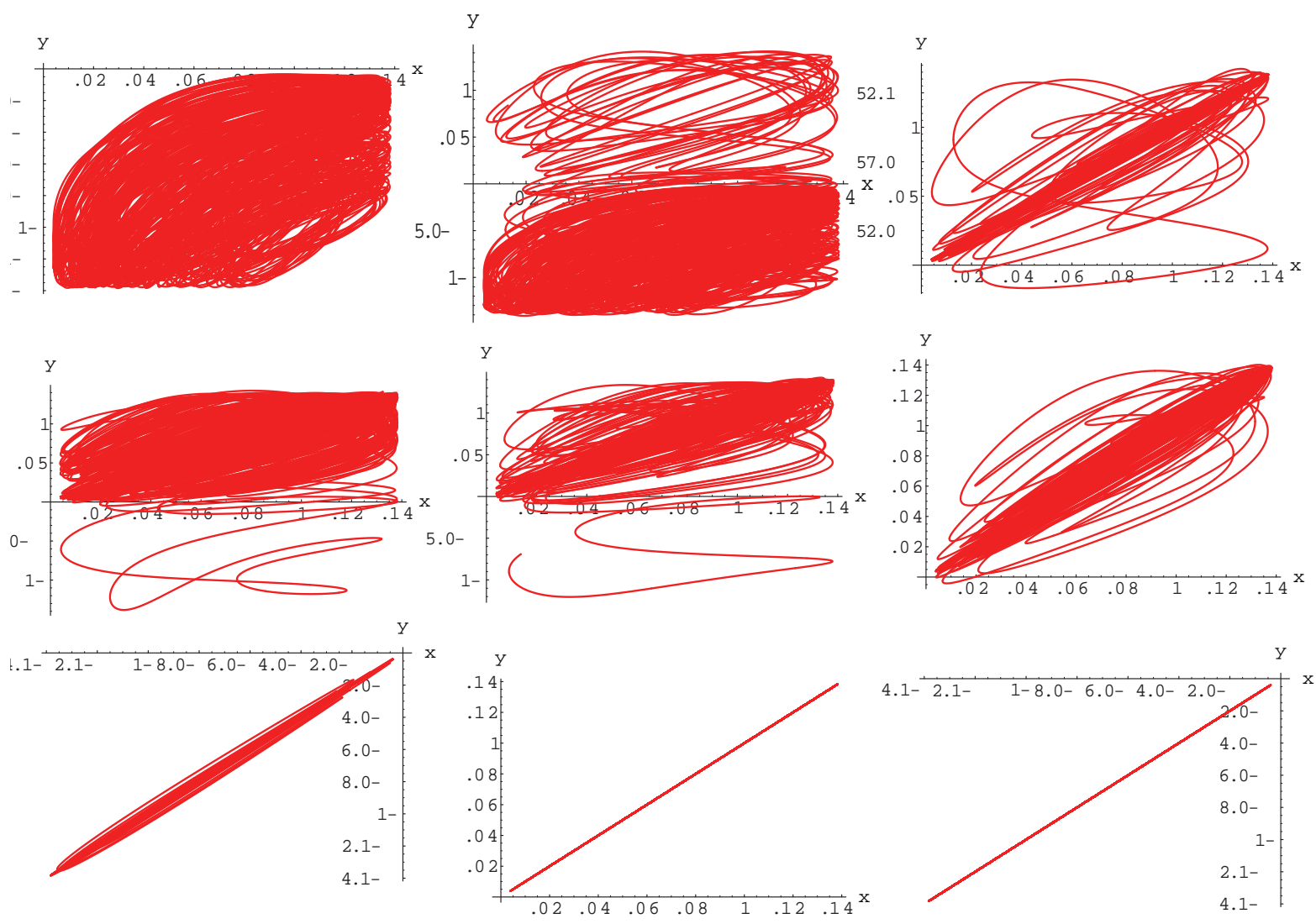

Fig. 11. Evolution of $x$ versus $y$ for the unimodal case in the unidirectional coupled Duffing oscillators $(\alpha=0.4, \beta=0.3578)$ for some values of $k: 0.003,0.005,0.022,0.103,0.111,0.12,0.136,0.195$ and 0.306 .

Table 1. Topological entropy, kneading determinant and sequences for $x$ for some values of the coupling parameter

\begin{tabular}{|c|c|c|c|}
\hline$k$ & $S_{x}$ & $D(t)$ & $h_{\text {top }}$ \\
\hline 0 & $(C R L R R R)^{\infty}$ & $\frac{(-1+t)\left[\left(-1+t^{2}\right)+t^{4}\right]}{1-t^{12}}$ & $0.24061 \ldots$ \\
\hline 0.05 & $(C R L R)^{\infty}$ & $\frac{-(1+t)\left(-1+t^{2}\right)}{1-t^{8}}$ & 0 \\
\hline 0.13 & $(C R L R R R L R L R)^{\infty}$ & $\frac{(-1+t)\left[\left(-1+t^{2}\right)\left(1-t^{4}\right)+t^{8}\right]}{1-t^{20}}$ & $0.20701 \ldots$ \\
\hline 0.5 & $(C R L R R R L R L R)^{\infty}$ & $\frac{(-1+t)\left[\left(-1+t^{2}\right)\left(1-t^{4}\right)+t^{8}\right]}{1-t^{20}}$ & $0.20701 \ldots$ \\
\hline 0.301 & $(C R L R R R L R L R)^{\infty}$ & $\frac{(-1+t)\left[\left(-1+t^{2}\right)\left(1-t^{4}\right)+t^{8}\right]}{1-t^{20}}$ & $0.20701 \ldots$ \\
\hline
\end{tabular}



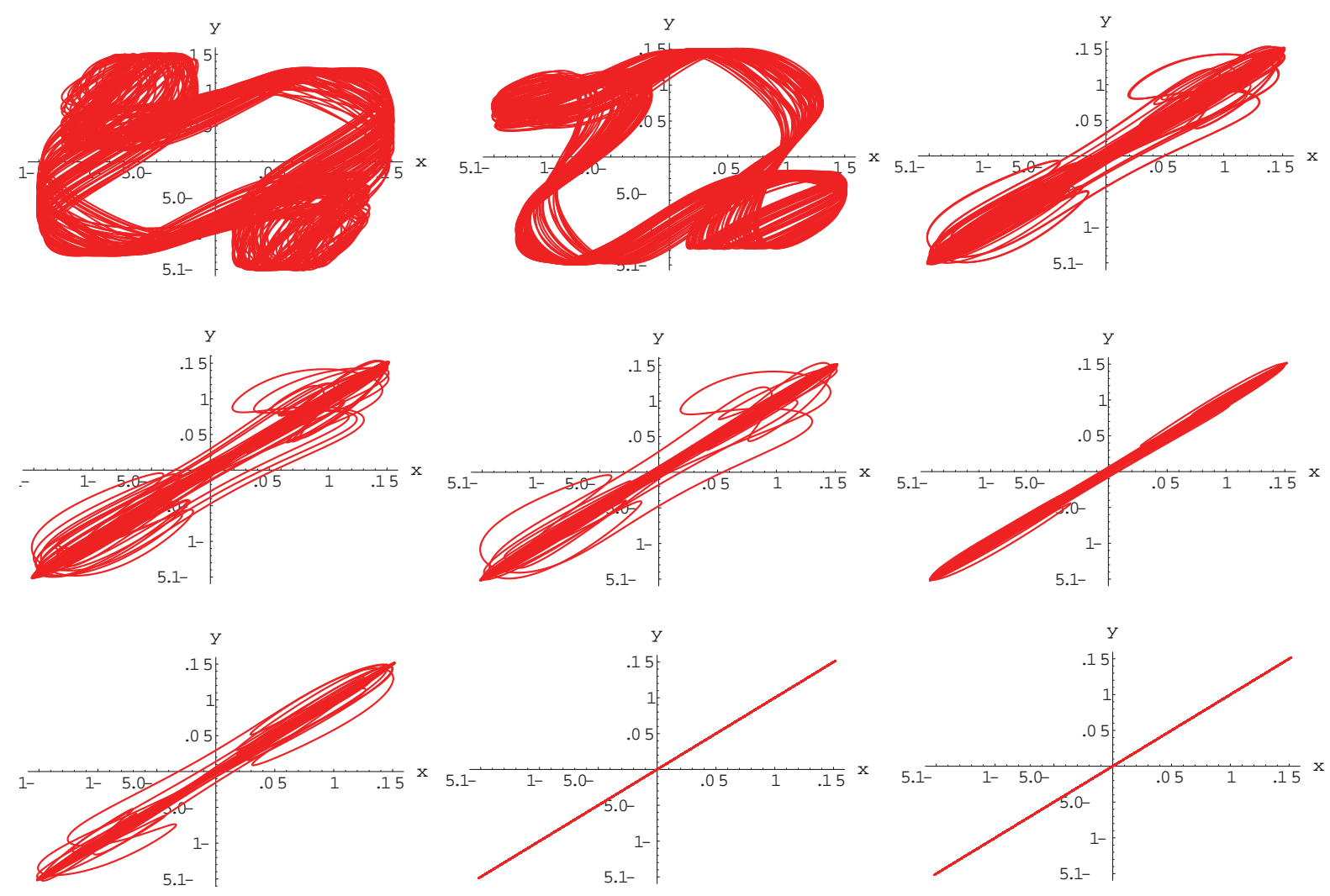

Fig. 12. Evolution of $x$ versus $y$ for the bimodal case in the unidirectional coupled Duffing oscillators $(\alpha=0.5, \beta=0.719)$ for some values of $k$ : $0.003,0.014,0.08,0.095,0.1,0.113,0.126,0.875$ and 0.916 .
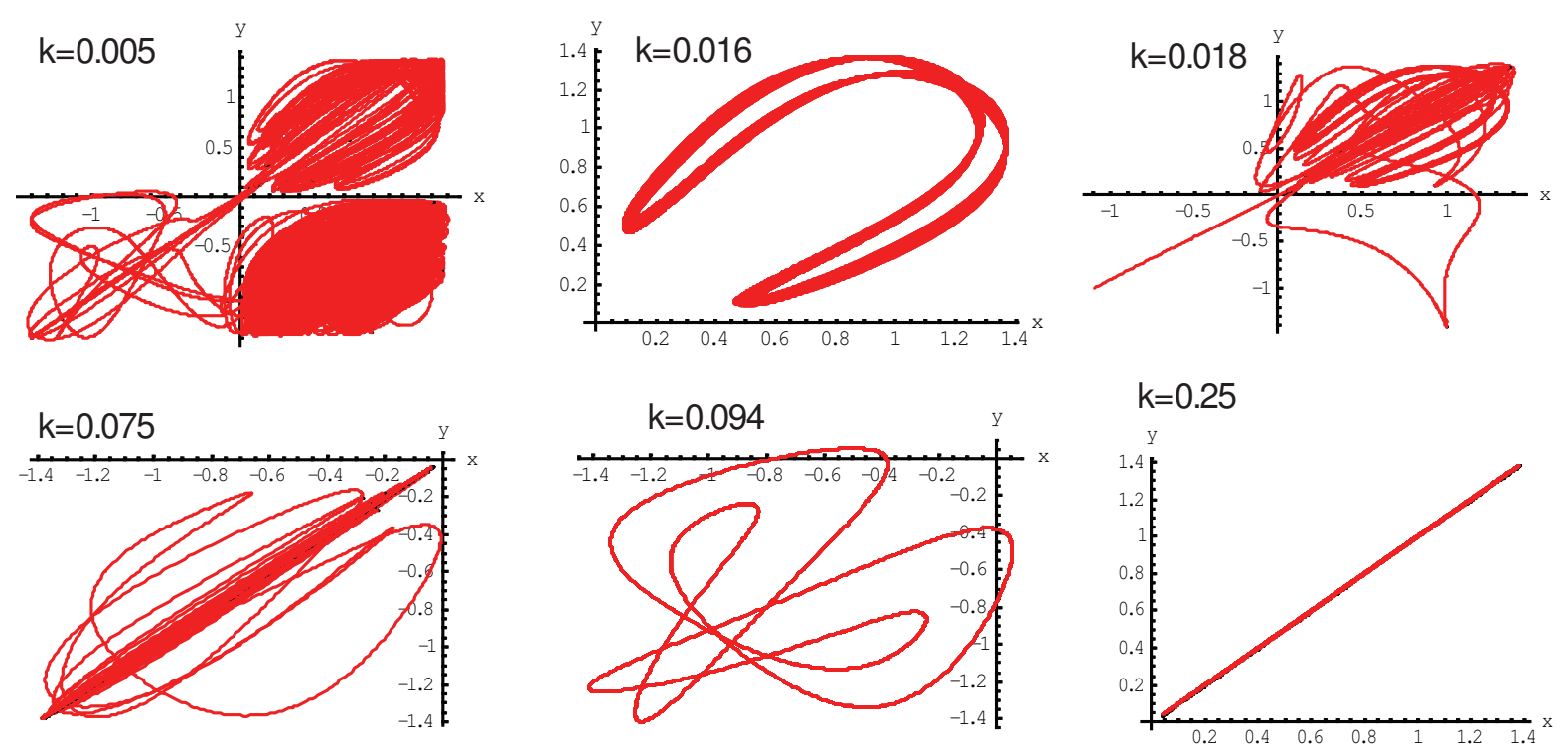

Fig. 13. Evolution of $x$ versus $y$ for the bidirectional coupled Duffing oscillators, for some values of $\mathrm{k}$, in the unimodal case $(\alpha=0.4, \beta=0.3578)$. 
$\left\{\begin{aligned} x^{\prime \prime}(t)= & x(t)-x^{3}(t)-\alpha x^{\prime}(t)-k[x(t)-y(t)] \\ & +\beta \cos (w t) \\ y^{\prime \prime}(t)= & y(t)-y^{3}(t)-\alpha y^{\prime}(t)+k[x(t)-y(t)] \\ & +\beta \cos (w t)\end{aligned}\right.$

We choose, for example, $w=1.18, x_{0}=0.5$, $x_{0}^{\prime}=-0.3, y_{0}=0.9, y_{0}^{\prime}=-0.2$ and $\alpha=0.4, \beta=$ 0.3578 , for the unimodal case and $\alpha=0.5, \beta=$ 0.719 , for the bimodal case.

See in Fig.13 the evolution of $x$ versus $y$ for the unimodal case. Although not shown in this figure, the graphics of the difference $y-x$ for $k$ greater then 0.122 are always a diagonal like in the picture for $k=0.25$, showing that these Poincaré unimodal maps are synchronized. For $\alpha=0.4$ and $\beta=0.3578$ we have $h_{\text {top }}=0.2406 \ldots$, then $s=1.272 \ldots$ If the coupled maps where piecewise linear maps with slope $s= \pm 1.272$, the synchronization will occurs for $c>c^{*}=$ $\frac{s-1}{2 s}=0.107$ and we see numerically that these unimodal Poincaré maps for the Duffing equations synchronizes at a little greater value, $k^{*} \approx 0.122$, so these pictures confirms numerically the above theoretical results, though we cannot guarantee that the semiconjugation between the unimodal and the piecewise linear maps is a conjugation.

\section{Conclusions and open problems}

We obtained explicitly the value $k^{*}$ of the coupling parameter, such that for $k>k^{*}$ two piecewise linear maps, unidirectional or bidirectional coupled are synchronized. Moreover we prove that, in certain conditions, the synchronization of two $m$-modal maps is equivalent to the synchronization of the corresponding conjugated piecewise linear maps, but for different values of the coupling parameter.

By a theorem from [Milnor \& Thurston, 1988] and [Parry, 1964] it is known that every $m$-modal map $f: I=[a, b] \subset R \rightarrow I$, with growth rate $s$ and positive topological entropy $h_{\text {top }}\left(\log s=h_{\text {top }}(f)\right)$ is topologically semi-conjugated to a $p+1$ piecewise linear map $T$, with $p \leq m$, defined on the interval $J=[0,1]$, with slope $\pm s$ everywhere and $h_{\text {top }}(T)=$ $h_{\text {top }}(f)=\log s$.

The maps $f: I \rightarrow I$ and $T: J \rightarrow J$ are semi-conjugated if there exist a function $h$ continuous, monotone and onto, $h: I \rightarrow J$, such that $T \circ h=h \circ f$.

If, in addition, $h$ is a homeomorphism, then $f$ and $T$ are said topologically conjugated.

We proved that in the case of topological conjugacy the synchronization of the two piecewise linear maps $T$ implies the synchronization of the two conjugated $m$-modal maps $f$. Furthermore, by a result of [Preston, 1989], (see also [Alves et al, 2005]), if $f$ is topologically transitive, then the mentioned semi-conjugacy is in fact a conjugacy.

By [Blokh, 1982] (see also [Alves et al, 2005]), we know that $h_{\text {top }}(f) \geq(1 / 2) \log 2$ holds for any topologically transitive map of the interval, but we observed numerically that in the case of coupled Duffing equations, the synchronization occurs for entropy values some how less than $(1 / 2) \log 2$, so it remains an open problem to find sufficient conditions for a semi-conjugacy to be a conjugacy.

The study and conclusions about synchronization of piecewise linear unimodal and bimodal maps, expressed in theorems 2.1 and 2.2, can be applied to guarantee the synchronization of more general maps.

\section{References}

Alves, J., Fachada, J. \& Ramos, J. S. [2005] "Detecting topological transitivity of piecewise monotone interval maps", Topology Appl., 153, 680-697.

Caneco, A., Fernandes, S., Grácio, C. \& Rocha, J. L. [2007] "Symbolic dynamics and networks", Proceedings of the International Workshop on Nonlinear Maps and Applications (NOMA'07), 42-45.

Caneco, A., Grácio, C. \& Rocha, J. L. [2008] "Symbolic dynamics and chaotic synchronization in coupled Duffing oscillators", J. Nonlinear Math. Phys., 15, 3, 102-111.

Caneco, A., Grácio, C. \& Rocha, J. L. [2009] "Kneading theory analysis of the Duffing equation", Chaos Solit. Fract., (in press).

Belykh, I., Hasler, M. \& Belykh, V. [2007] "When symmetrization guarantees synchronization in directed networks", Int. J. Bif. Chaos, 17 (10). 
Blokh, A. [1982] "On sensitive mappings of the interval", Russian Math. Surveys 37 (2), 203-204.

Boccaletti, S., Kurths, J., Osipov, G., Valladares, D.L. \& Zhou, C. S. [2002] "The synchronization of chaotic systems", Physics Reports, 366, 1-101.

Cao, J., \& Lu, J. [2006] "Adaptive synchronization of neural networks with or without time-varying delays", Chaos, 16, 013133 (6pp).

Chazottes, J.-R., Ramirez, L. \& Ugalde, E. [2005] "Finite type approximations of Gibbs measures on sofic subshifts", Nonlinearity, 18, 445-463.

Gonzlez-Miranda, J. M. [2004] Synchronization and Control of Chaos. An introduction for scientists and engineers, Imperial College Press.

Kenfack, A. [2003] "Bifurcation structure of two coupled periodically driven double-well Duffing oscillators", Chaos Solit. Fract., 15, 205-218.

Kyprianidis, I. M., Volos, C., Stouboulos, I. N. \& Hadjidemetriou [2006] "J. Dynamics of two resistively coupled Duffing-type electrical oscillators", Int. J. Bif. Chaos 16, 1765-1775.

Lampreia, J. P. \& Ramos, J. S. [1997] "Symbolic Dynamics for Bimodal Maps", Portugaliae Math. 54 (1), 1-18.

Luo, A. C. J. [2008] "Global tangency and transversality of periodic flows and chaos in a periodically forced, damped Duffing oscillator", Int. J. Bif. Chaos, 18, (1), 1-49.

Milnor, J. \& Thurston, W. [1988] "On iterated maps of the interval", Lect. Notes in Math, 1342, Springer-Verlag, 465-563.

Misiurewicz, M. \& Szlenk, W. [1980] "Entropy of piecewise monotone mappings", Studia Math. 67, 45-63.

Parry, W. [1964] "Symbolic dynamics and transformations of the unit interval", Trans. Amer. Math. Soc., 122, 368-378.

Pecora, L. M. \& Carroll, T. L. [1990] "Synchronization in chaotic systems", Phys. Rev. Lett. 64, 821-824.

Pecora, L. M. \& Carroll, T. L. [1991] "Driving systems with chaotic signals", Phys. Rev. A 44, 2374-2383.

Pikovsky, A., Rosenblum, M. \& Kurths, J. [2001] Synchronization: a universal concept in nonlinear sciences, Cambridge University Press.
Preston, C. [1989] "What you need to knead", $A d v$. in Math. 78, 192-252.

Rocha, J. L. \& Ramos, J. S. [2006] "Computing conditionally invariant measures and escape rates", Neural, Parallel \& Sc. Comput., 14, 97-114.

Shuai, J.W., Wong, K.W. \& Cheng, L.M. [1997] "Synchronization of spatiotemporal chaos with positive conditional Lyapunov exponents", Phys Rev E 56, 2272.

Vincent, U. E. \& Kenfack, A. [2008] "Synchronization and bifurcation structures in coupled periodically forced non-identical Duffing oscillators", Phys. Scr., 77, 045005 (7pp). 